\title{
2105 An analysis of failure in thin coatings subjected to contact loading
}

\author{
S. Rash Ahmadi*, M. Fujii**, M. Shakeri*, A. Sadough*, T. Shirakawa** \\ *Department of Mechanical Engineering, Amirkabir University of Technology, \\ 424 Hafez Avenue, Tehran, Iran \\ ** Graduate School of Natural Science and Technology, Okayama University, Okayama, Japan
}

\begin{abstract}
Hard coatings, which are used to protect surfaces that are subjected to contact loading, may fracture or delaminate from their substrates. The probability of the extension of surface crack due to cylindrical indentation is analyzed. The coated half-space is loaded by a cylindrical indenter, which slides over the surface of the coating. Contact loading problems typically result in multiaxle and inhomogeneous stress fields near the contact surface. This behaviour which strongly affects the stress intensity factors is taken into account in the failure analysis. A suitable method is described for calculating the contact stress fields in coating and substrate. Edge cracks are selected to model the surface flaws. The coating is idealized as a brittle-elastic layer of uniform thickness, which is bonded to the surface of a half space. Method of linear elastic fracture mechanics are then used to calculate the loads to initiate fracture in the coating.
\end{abstract}

Kew words: Tribology, Coating, Contact, Fracture

\section{INTRODUCTION}

During the last decades coatings have played an increasingly important role in mechine industry, primarily utilizing their high resistance. They are often used to protect surfaces that are snbjected to contact loading. In all these applications, failure of coating is a concern. There are several different mechanisms of failure in coated components, such as excessive plastic deformation, rolling coantact fatigue, or various forms of wear. This type of damage can often be prevented by using a hard material for the coating. The high hardness of these materials is obtained at the expense of a low fracture toughness. Consequently, fracture and delamination of the film become the dominant failure mechanism in many practical situations.

The resistance of the coating to contact fracture is frequently measured using the"scratch test". The test involves sliding a conical or spherical indenter over the surface, while subjecting the indenter to a progressively increasing load. The critical load required to initiate fracture in the film or to cause the film to delaminate from the substrate gives the measure of the strength of the coating or interface. Tests of this type are useful to rank the relative strengths of various coated solids, but provide little insight into the influence of material properties or loading conditions on the failure mechanism. Some of these limitations would be overcome by a detailed analysis of the scratch test, and some progress in this direction has been made $[1,2]$. However, data obtained from scratch tests remain a qualitative rather than quantative measure of the strength of coating.

An alternative approach is to analyse in detail the deformation induced by the contact load and to predict the strength of the coated solid in terms of measurable properties of the materials. Most studies of contact fracture have considered the behaviour of uncoated brittle solids.

The objective in this paper is to analyse in detail the probability of the extension of surface crack due to cylindrical indentation illustrated in Fig. 1, with radius $R$.

The authors suppose that the indenter is elastic, with Young's modulus $E_{i}$ and Poission's ratio $v_{i}$. Coulomb friction, with friction coefficent $f$, is assumed to act between the indenter and the surface of the coating, so that the half-space is subjected to the distribution of traction $q(x)=f . p(x)$ acting in direction of motion of the indenter.

\section{THE CONTACT PROBLEM FOR THE HALF- SPACE}

The problem of elastic coatings of uniform thickness perfectly bounded to an underlying dissimilar elastic halfplane is investigated on the basis of two dimensional theory of elasticity. Under the conditions of plane strain, the stress components $\sigma_{x x}, \sigma_{y y}, \sigma_{x y}$ can be expressed as [3, 4]:

$$
\sigma_{x x}=\frac{\partial^{2} \phi}{\partial y^{2}}, \sigma_{y y}=\frac{\partial^{2} \phi}{\partial x^{2}}, \sigma_{x y}=-\frac{\partial^{2} \phi}{\partial x \partial y}
$$

where $\phi$ is Airy stress function. It must be biharmonic $\nabla^{4} \phi=0$. Solution of the equation $\nabla^{4} \phi=0$ can be chosen in the form [5]:

$\phi=\int_{0}^{\infty}\left[\left(A_{1}+A_{2} y\right) e^{-w y}+\left(A_{3}+A_{4} y\right) e^{w y}\right]\left(c_{1} \cos w x+c_{2} \sin w x\right) d w$

where $A_{1}, A_{2}, \ldots, c_{1}, c_{2}$ are parameters which are only related to parameter $w$. Writing $Q_{1}$ for $\nabla^{2} \phi$ which represent $\sigma_{x}+\sigma_{y}$ we observe that $Q_{1}$ is a harmonic function, and will have a conjugate harmonic function $Q_{2}$.

The stresses and displacements for the plane strain condition can be written as [5]:

$$
u=\frac{1+v}{E}\left[4(1-v) q_{1}-\frac{\partial \phi}{\partial x}\right], v=\frac{1+v}{E}\left[4(1-v) q_{2}-\frac{\partial \phi}{\partial y}\right]
$$




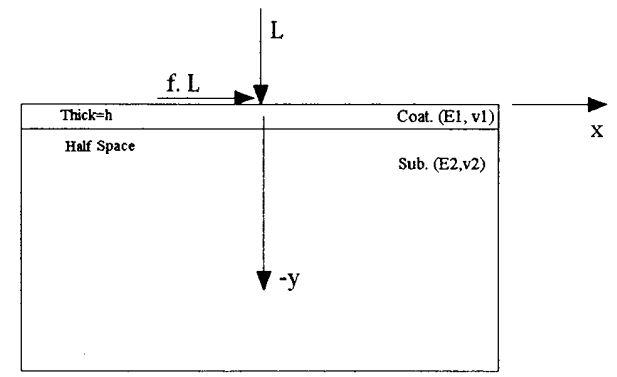

Fig.1: Coated half-space subjected to plain strain contact

$$
\sigma_{x x}=\frac{\partial^{2} \phi}{\partial y^{2}}, \sigma_{y y}=\frac{\partial^{2} \phi}{\partial x^{2}}, \sigma_{x y}=-\frac{\partial^{2} \phi}{\partial x \partial y}
$$

where $\frac{\partial q_{1}}{\partial x}=\frac{1}{4} Q_{1}$ and $\frac{\partial Q_{1}}{\partial x}=\frac{\partial Q_{2}}{\partial y}, \frac{\partial Q_{1}}{\partial y}=-\frac{\partial Q_{2}}{\partial x}$

a) Normal load: In view of the symmetry of the normal load and the requirement that the substrate must be stressfree at large distance from loading point, Airy stress functions for coatings and substrate can be written as [5]:

$$
\begin{gathered}
\phi^{I}=\int_{0}^{\infty}\left[\left(A_{1}+A_{2} y\right) e^{-w y}+\left(A_{3}+A_{4} y\right) e^{w y}\right] \cos w x d w \\
\phi^{I I}=\int_{0}^{\infty}\left[\left(A_{5}+A_{6} y\right) e^{w y}\right] \cos w x d w,
\end{gathered}
$$

where $A_{j}$ are general functions of Fourier transform variable $w$, subscripts I, II refer to coating and substrate respectively.

The stress and displacement fields for the coatings and substrate can now be derived.

The unknown factions $A_{i}(w)$ are now determined from the boundary conditions at the surface:

$$
y=0 \rightarrow\left\{\begin{array}{l}
\sigma_{y y}=-p(x) \\
\sigma_{x y}=0
\end{array}\right.
$$

where $p(x)$ is,

$$
\begin{aligned}
& p(x)= \begin{cases}p_{\max } \sqrt{1-(x / a)^{2}} & \mid \begin{array}{l}
|x| \leq a \\
0
\end{array} \\
|x| \geq a\end{cases} \\
& \text { and } p_{\max }=\frac{2 L}{\pi a}, \quad a=\sqrt{\frac{8}{\pi} \cdot \frac{R}{E^{\prime}} \cdot L}, \quad \frac{2}{E^{\prime}}=\frac{1-v_{i}^{2}}{E_{i}}+\frac{1-v_{1}^{2}}{E_{1}}
\end{aligned}
$$

where $L$ is normal load per unit length, $E_{i}$ and $E_{1}$ are Young's modulus of indenter and coating respectively, $R$ is indenter radius and $a$ is contact radius.

The continuity conditions of stress and displacements along the interfaces are:

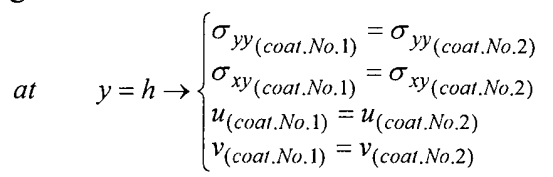

b) Tangential load: Similar to the argument of the previous section, Airy stress functions for coatings and substrate can be written in the form [5]:

$$
\begin{aligned}
& F^{I}=\int_{0}^{\infty}\left[\left(A_{1}+A_{2} y\right) e^{-w y}+\left(A_{3}+A_{4} y\right) e^{w y}\right] \sin w x d w \\
& F^{I I}=\int_{0}^{\infty}\left[\left(A_{5}+A_{6} y\right) e^{w y}\right] \sin w x d w
\end{aligned}
$$

Using the procedure described in the previous section, the stress and displacement field for the coatings and substrate can be found:
In this case the boundary conditions at the surface are as follows:

$$
y=0 \rightarrow\left\{\begin{array}{l}
\sigma_{y y}=0 \\
\sigma_{x y}=-f \cdot p(x)=q(x)
\end{array}\right.
$$

These conditions apply in equation (32), where $q(x)$ is:

$$
q(x)= \begin{cases}-f \cdot p_{\max } \sqrt{1-(x / a)^{2}} & |x| \leq a \\ 0 & |x| \geq a\end{cases}
$$

The continuity conditions of stress and displacements along the interfaces are similar to (7).

The unknown factions $A_{j}(w)$ are now determined from the boundary and continuity conditions.

c) Total stress and displacement fields: The total stress and displacement fields are obtained from superposing the results of normal and tangential forces, so we have [5]:

$$
\begin{aligned}
& \sigma_{x x}=\sigma_{x x}{ }^{n} \cdot L+T \cdot \sigma_{x x}{ }^{\prime} \\
& \sigma_{y y}=\sigma_{y y}{ }^{n} \cdot L+T \cdot \sigma_{y y}{ }^{t} \\
& \sigma_{x y}=\sigma_{x y}{ }^{n} \cdot L+T \cdot \sigma_{x y}{ }^{\prime} \\
& u=u^{n} \cdot L+T \cdot u^{t} \\
& v=v^{n} \cdot L+T \cdot v
\end{aligned}
$$

where $n$ and $t$ refer to the solution of normal and tangential load respectively.

In order to find the numerical results the material properties illustrate in Table 1 are used [5].

The numerical in TiN/St. system for $L=10 \mathrm{kN} / \mathrm{m}$, $f=0.08$ and $3 \mu \mathrm{m}$ coating thickness in $y=1 \mu \mathrm{m}$ from surface have been shown in Fig. 2. In Fig. 3 distribution of $\sigma_{x x}$ has been presented during thickness of coating.

Table 1: Material properties used in analytic solution
\begin{tabular}{|c|c|c|}
\hline Section & Coating(TiN) & Substrate(St.) \\
\hline$E$ & $386 \mathrm{GPa}$ & $207 \mathrm{GPa}$ \\
\hline$v$ & 0.22 & 0.3 \\
\hline
\end{tabular}

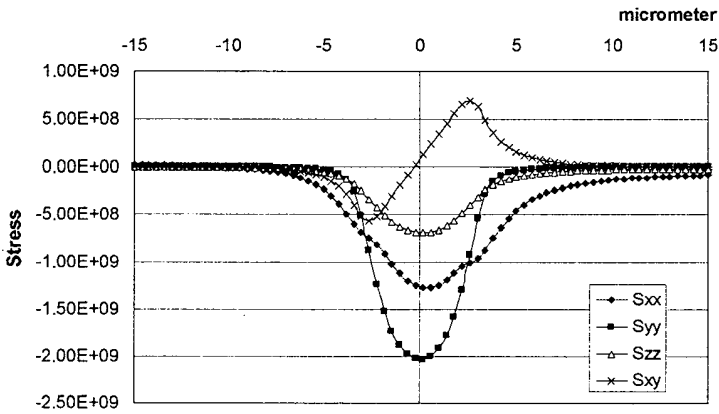

Fig. 2: Stress distribution in TiN/St. system for $L=10 \mathrm{kN} / \mathrm{m}$, $f=0.08$, thick. $=3 \mu \mathrm{m}$ and in $y=-1 \mu \mathrm{m}$.

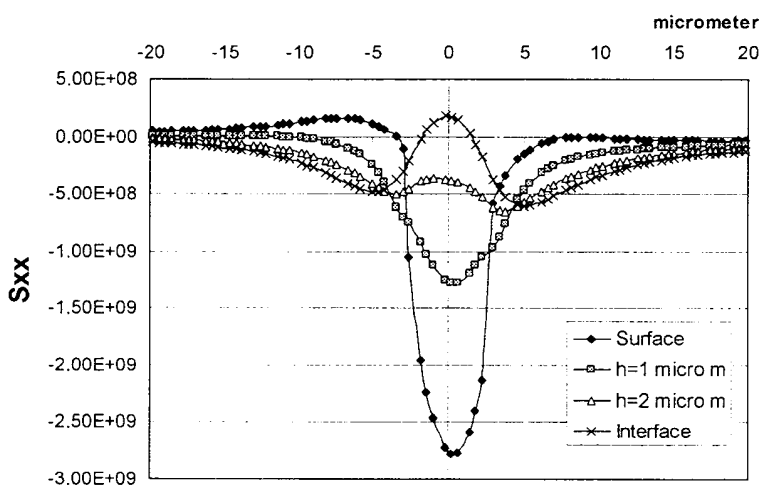

Fig. 3: $\sigma_{x x}$ during thickness of coating in TiN/St. system for $L=10 \mathrm{kN} / \mathrm{m}, f=0.08$, thick. $=3 \mu \mathrm{m}$ 


\section{FRACTURE MECHANICS OF COATED SOLID}

The next objective is to predict the conditions necessary for fracture to occur in the coated half-space. We begin by outlining the main assumptions that we have made in the analysis. Edge cracks are selected to model the surface flaws. We follow standard procedures to analyze fracture of the coating. The state of stress near the tip of a micro crack is assumed to be characterized by mode I and mode II stress intensity factor $K_{I}$ and $K_{I I}$. If the stress intensity factor at the tip of micro crack exceed a critical combination, the crack will propagate. Here, we have used the intensity of the greatest tensile stress as a fracture criterion, and used the plane strain fracture toughness $K_{I C}$ to parameterize the fracture resistance of the solid. The effective stress intensity factor defined as [7]:

$$
K_{\text {eff }}=\sqrt{K_{I}^{2}+K_{I I}^{2}}
$$

According to the maximum stress criterion, a crack under mixed-mode loading starts to propagate when $K_{\text {eff }} \geq K_{I C}$.

The solution begins by calculating the contact stress fields in the untracked solid. We assume that the contact pressure remains Hertzian, so that the contact loading may be parameterized by the peak pressure $p_{\max }$, the contact radius $a$, the surface traction coefficient $f$. We denote the contact stress field by $\tilde{\sigma}_{i j}(x, y)$.

Consider a crack of length $c$ in the coating normal to surface behind the indenter as illustrated in Fig 4. The behavior of material in the process zone, and hence the critical loading required to cause fracture, is assumed to be characterized by stress state some distance from the crack tip. Wherever a crack is pulled open by the load, its faces must be free of tractions. Denote the normal and shear stresses along the crack-line by $N(y)$ and $S(y)$ respectively. When the crack open we have:

$$
N(y)=S(y)=0
$$

The contact stress fields must then be corrected for the presence of the cracks.

As mentioned before, $\tilde{\sigma}_{i j}(x, y)$ is the stress field induced in the material in the absence of crack.

Now consider separately the problem of continuum of material, geometrically identical to that subject to the remote bulk load, but now devoid of any external loading, Fig. 5(c). Let the crack faces be forced to separate by the insertion of additional material. The stress state, is thereby induced in the material, in particular, the tractions induced along the line of crack.

It follows from the superposition principle that the resultant state of stress is obtained by a simple addition of the solutions to these two stresses:

$$
\sigma_{i j}(x, y)=\tilde{\sigma}_{i j}(x, y)+\bar{\sigma}_{i j}(x, y)
$$

Provided that the boundary conditions along the traction-free crack faces are satisfied.

The corrective stresses $\bar{\sigma}_{i j}(x, y)$ may be generated by distributing dislocations continuously along crack. To obtain the expression for the stresses, we used the procedure presented by Hills, et al. [8]. So:

$$
\begin{aligned}
& N(y)=\tilde{\sigma}(y)+\frac{2 \mu}{\pi(\kappa+1)} \int_{0}^{c} B_{x}(\xi)\left[\frac{1}{x-\xi}+K^{\prime}(y ; \xi)\right] d \xi \\
& S(y)=\tilde{\tau}(y)+\frac{2 \mu}{\pi(\kappa+1)} \int_{0}^{c} B_{y}(\xi)\left[\frac{1}{x-\xi}+K^{\prime}(y ; \xi)\right] d \xi
\end{aligned}
$$

where the kernel $K^{\prime}(y ; \xi)$ is:

$$
K^{\prime}(y ; \xi)=-\frac{1}{y+\xi}-\frac{2 \xi}{(y+\xi)^{2}}+\frac{4 \xi^{2}}{(y+\xi)^{3}}
$$

and $\kappa=3-4 v$ and $\mu=\frac{E}{2(1+2 v)}$.

The more direct way of handling the problem is to put the integral equation in normalized form

$$
s=2 \xi / c-1, t=2 y / c-1
$$

With rewriting $K^{\prime}(y ; \xi)$ in normalized coordinates:

$$
K^{\prime}(t, s)=\frac{2}{c}\left[-\frac{1}{s+t+2}-\frac{2(s+1)}{(s+t+2)^{2}}+\frac{4(s+1)^{2}}{(s+t+2)^{3}}\right.
$$
follows:

Base the procedure of Hills, et al. [8] we can find as

$$
\begin{gathered}
-\frac{K+1}{2 \mu} \tilde{\sigma}\left(t_{k}\right)=\sum_{i=1}^{N} \frac{2\left(1+s_{i}\right)}{2 N+1} \phi_{x}\left(s_{i}\right)\left[\frac{1}{t_{k}-s_{i}}+\frac{c}{2} K^{\prime}\left(t_{k}, s_{i}\right)\right] \\
-\frac{K+1}{2 \mu} \tilde{\tau}\left(t_{k}\right)=\sum_{i=1}^{N} \frac{2\left(1+s_{i}\right)}{2 N+1} \phi_{y}\left(s_{i}\right)\left[\frac{1}{t_{k}-s_{i}}+\frac{c}{2} K^{\prime}\left(t_{k}, s_{i}\right)\right] \\
k=1 \ldots N
\end{gathered}
$$

where $s_{i}=\cos \left(\pi \frac{2 i-1}{2 N+1}\right), t_{k}=\cos \left(\pi \frac{2 k}{2 N+1}\right), i, k=1 \ldots . . N$

In this case we have the same number of equations as unknowns.

Finally $K_{I}$ and $K_{I I}$ are as follows:

$$
K_{I, I I}=\sqrt{\pi a} \frac{2 \mu}{\pi(\kappa+1)} \sqrt{2} \phi_{x, y}(+1)
$$

The value of $\phi_{x}(+1)$ and $\phi_{y}(+1)$ can be obtained as follow [7]:

$$
\phi(+1)=M_{E}(+1) \sum_{i=1}^{N} \phi_{E}(+1) \phi\left(s_{i}\right)
$$

where $M_{E}(+1)=\frac{2}{2 N+1}$ and

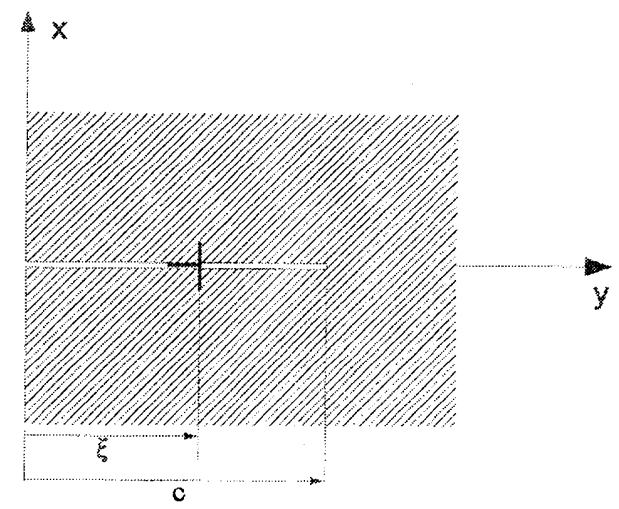

Fig.4: Normal surface-breaking crack within coating. A typical dislocation is shown at $y=\xi$ 


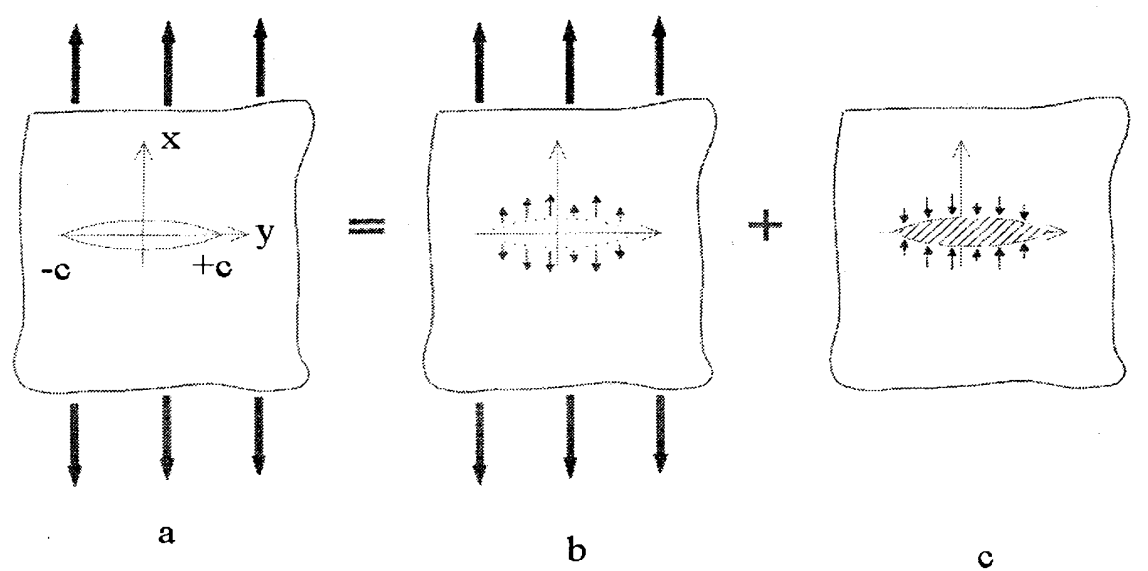

Fig.5: a) overall problem b) stress in perfect body c) separated material

$$
\phi_{E}(+1)=\cot \left[\frac{2 i-1}{2 N+1} \frac{\pi}{2}\right] \sin \left[\frac{2 i-1}{2 N+1} N \pi\right]
$$

In order to show the numerical results, the stress intensity factors for TiN/St. system with $3 \mu \mathrm{m}$ thickness of coating and subjected to $10 \mathrm{kN} / \mathrm{m}, 20 \mathrm{kN} / \mathrm{m}$ and $100 \mathrm{kN} / \mathrm{m}$ cylindrical loads, are presented. The material properties illustrated in table 1 are used for calculation. Crack length has been assumed to be $0.5 \mu \mathrm{m}$. Contact parameters can be obtained from (6) and have been presented in Table 2.

Table 2: contact parameters used in analytic solution

\begin{tabular}{|c|c|c|c|}
\hline \multicolumn{5}{|c|}{ TiN/St., cylindrical load } \\
\hline Load $[\mathrm{N} / \mathrm{m}]$ & 10000 & 20000 & 100000 \\
\hline $\mathrm{R} 1[\mathrm{~m}]$ & 0.0002 & 0.0002 & 0.0002 \\
\hline $\mathrm{R} 2[\mathrm{~m}]$ & $1 \mathrm{E}+100$ & $1 \mathrm{E}+100$ & $1 \mathrm{E}+100$ \\
\hline $\mathrm{E} 1[\mathrm{~Pa}]$ & $1.141 \mathrm{E}+12$ & $1.141 \mathrm{E}+12$ & $1.141 \mathrm{E}+12$ \\
\hline $\mathrm{E}^{2}[\mathrm{~Pa}]$ & $3.86 \mathrm{E}+11$ & $3.86 \mathrm{E}+11$ & $3.86 \mathrm{E}+11$ \\
\hline$v 1$ & 0.07 & 0.07 & 0.07 \\
\hline$v 2$ & 0.22 & 0.22 & 0.22 \\
\hline $\mathrm{R}[\mathrm{m}]$ & 0.0002 & 0.0002 & 0.0002 \\
\hline $\mathrm{E}^{\prime}[\mathrm{Pa}]$ & $5.993 \mathrm{E}+11$ & $5.993 \mathrm{E}+11$ & $5.993 \mathrm{E}+11$ \\
\hline $\mathrm{a}[\mathrm{m}]$ & $2.915 \mathrm{E}-06$ & $4.123 \mathrm{E}-06$ & $9.219 \mathrm{E}-06$ \\
\hline $\mathrm{Pmax}[\mathrm{Pa}]$ & $2.184 \mathrm{E}+09$ & $3.088 \mathrm{E}+09$ & $6.906 \mathrm{E}+09$ \\
\hline$a[\mu \mathrm{m}]$ & 2.915 & 4.123 & 9.219 \\
\hline$P \max [\mathrm{MPa}]$ & 2183.760 & 3088.304 & 6905.657 \\
\hline
\end{tabular}

The effective stress intensity factor for $10 \mathrm{kN} / \mathrm{m}$ is $88642 \mathrm{Pam}^{1 / 2}$, and for $20 \mathrm{kN} / \mathrm{m}$ and $100 \mathrm{kN} / \mathrm{m}$ are $132963 \mathrm{Pam}^{1 / 2}$ and $281881 \mathrm{Pam}^{1 / 2}$ respectively. The deatails of stress intensity factors have been shown in Table 3 . These results show that mode $I$ is dominant mode.

Table 3: Stress Intensity Factors for different loads

\begin{tabular}{|c|c|c|c|}
\hline \multicolumn{4}{|c|}{ Stress Intensity Factor } \\
\hline Load [N/m] & 10000 & 20000 & 100000 \\
\hline$K_{I}\left[\mathrm{Pam}^{1 / 2}\right]$ & 88641 & 132962 & 281880.9 \\
\hline$K_{I I}\left[\mathrm{Pam}^{1 / 2}\right]$ & 73.6 & 160.4 & 341.1 \\
\hline$K_{\text {cff }}\left[\mathrm{Pam}^{1 / 2}\right]$ & 88642 & 132963 & 281881 \\
\hline
\end{tabular}

\section{CONCLUSIONS}

The mechanism of failure in a brittle layered solid loaded by sliding cylindrical contact investigated. According to this analysis, the conditions necessary to initiate fracture from vertical flaws in the layer can be calculated. A suitable method was described for calculating the contact stress fields in coating and substrate subjected to cylindrical contact and these inhomogeneous stress fields near the contact surface which strongly affects the stress intensity factors is taken into account in the failure analysis.

\section{REFERENCES}

[1] Venkataraman, S., Kohlstedt, D. K. and Gerberich, W. W. , Continuous micro indentation measurements of metal-ceramic adhesive strengths, Thin Films: Stresses and Mechanical Properties III, Mater. Res. Symp. Proc. 239 (1989) 591-597.

[2] Venkataraman, S., Kohlstedt, D. K. and Gerberich, W. W., Metal-ceramic interfacial fracture resistance using continous microscratch technique, Thin Solid Films, 233 (1993) 269-275.

[3] Timoshenko, S.P., Theory of elasticity, Mc Graw Hill International Book Company, (1982).

[4] Muskhelishvili, N. I., Some Basic Problem of Mathematical Theory of Elasticity, Noordhoff international Publishing Leyden, (1975).

[5] Shakeri, M., Sadough, A. and Rash Ahmadi, S., Elastic stress analysis of bi-layered isotropic coatings and substrate subjected to line scratch indentation, J. Mat. Process. Technol. 196 (2008) 213-221.

[6] Stallard, J., Poulat, S. and Teer, D. G., The study of the adhesion of a TiN coating on steel and titanium alloy substrates using a multi-mode scratch tester, Tribology International xx (2006) 1-8.

[7] Licht, V., Hulsmeier, P. and Fett, T., Probability of cone crack initiation due to spherical contact loading, J. of the European Ceramic Society 24 (2004) 2907-2915.

[8] Hills, D. A., Kelly, P. A., Dai, D. N. and Korsunsky, A. M., Solution of Crack Problems, The Distributed Dislocatin Technique, Kluwar Academic Publishers (1996). 